\title{
Quantum cosmology with dynamical vacuum in a minimal-length scenario
}

\author{
M. F. Gusson ${ }^{1, \mathrm{a}}$, A. Oakes O. Gonçalves ${ }^{1,3, \mathrm{~b}}$, R. G. Furtado ${ }^{2, \mathrm{c}}$, J. C. Fabris ${ }^{1,4, \mathrm{~d}}$, J. A. Nogueira ${ }^{1, \mathrm{e}}$ \\ ${ }^{1}$ Universidade Federal do Espírito Santo-Ufes, Vitória, Espírito Santo 29.075-910, Brazil \\ ${ }^{2}$ Universidade Federal do Espírito Santo-Ufes, São Mateus, Espírito Santo 29.932-540, Brazil \\ ${ }^{3}$ Present Address: Instituto Federal Baiano, Campus Itapetinga, Itapetinga, BA, Brazil \\ ${ }^{4}$ National Research Nuclear University MEPhI, Kashirskoe sh. 31, Moscow 115409, Russia
}

Received: 23 December 2020 / Accepted: 2 April 2021 / Published online: 20 April 2021

(C) The Author(s) 2021

\begin{abstract}
In this work, we consider effects of the dynamical vacuum in quantum cosmology in presence of a minimum length introduced by the GUP (generalized uncertainty principle) related to the modified commutation relation $[\hat{X}, \hat{P}]:=\frac{i \hbar}{1-\beta \hat{P}^{2}}$. We determine the wave function of the Universe $\psi_{q p}(\xi, t)$, which is solution of the modified Wheeler-DeWitt equation in the representation of the quasiposition space, in the limit where the scale factor of the Universe is small. Although $\psi_{q p}(\xi, t)$ is a physically acceptable state it is not a realizable state of the Universe because $\psi_{q p}(\xi, t)$ has infinite norm, as in the ordinary case with no minimal length.
\end{abstract}

\section{Introduction}

The Standard Cosmological Model (SCM) is based on an expanding universe which has a very hot and dense origin. The extrapolation of the SCM for very early times leads to energy scales deep in the Planck regime and, strictly speaking, the expanding universe would have a singular initial state. On the other hand, in order to explain many features of the observed Universe today, a period of short but rapid, almost exponential, expansion, which is called inflationary phase, is necessary in the very early Universe. It is expected that in the primordial Universe quantum effects are relevant and a quantum approach is necessary to describe the gravitational effects. A quantum approach to gravity is also necessary in the study of black holes. The features of com-

\footnotetext{
a e-mail: michaelgusson@outlook.com

b e-mail: andre.oakes@gmail.com

c e-mail: raphafurtado@terra.com.br

d e-mail: julio.fabris@ cosmo-ufes.org

e e-mail: jose.nogueira@ufes.br (corresponding author)
}

pact astrophysical objects like neutron stars, and even white dwarfs, may be also affected by quantum gravity effects. However, we have no quantum gravity theory which is fully acceptable, although there are many proposals for it. An important aspect of almost all proposals for quantization of the gravity is the prediction of the existence of a minimal length. Thus, an effective description of the effects of quantum gravity through a phenomenological approach can be obtained by considering a minimal-length scenario [1-4]. Some results in the literature indicate that the introduction of a minimal length could also solve the problems like the initial singularity (big bang singularity) [5-13], the black hole complete evaporation (catastrophic evaporation of a black hole) [3,14-18], the cosmological constant [12,19-21], the Chandrasekhar limit $[4,18,20]$, the instability of the Einstein static universe (emergent universe) [22], the vanishing of cosmological constant in multiverse theories [19], and so on.

We can obtain a minimal-length scenario by modifying the Heisenberg uncertainty principle (HUP) between the position and momentum operators. There are several different proposals of modification of the HUP which introduce a minimal-length scenario. Those modified uncertainty principles are called Generalized Uncertainty Principle (GUP). ${ }^{1}$ GUP's have been derived from different contexts such as string theory [27-32], black hole physics [19,33-35] and extra dimensions [36].

Although the most common modified or deformed commutation relation (MCR) associated with a GUP (KMM GUP [37]) is quadratic in the momentum operator, effects of a MCR with a linear term in the momentum operator, which leads to a minimal length and a maximum momentum [38], have been studied in contexts of cosmological models

\footnotetext{
${ }^{1}$ Readers interested in finding out more about minimal-length literature may consult Refs. [17,23-26].
} 
[10,11,39-41]. Nevertheless, apparently this GUP leads to non-unitary theories [40] and it is not compatible with current data available [42].

In general, the inflationary phase is described by introducing a scalar field. Hence, in an inflationary quantum cosmology theory it is necessary to perform the second quantization of that scalar field. Consequently, in a minimal-length scenario that scalar field and its conjugate momentum have to obey the modified commutation relations [43-46]. As it is well known, the ordinary approach of the third quantization $^{2}$ of the Wheeler-DeWitt (WDW) equation leads to the vanishing of the cosmological constant. However, it might be possible to obtain a non-zero cosmological constant in a minimal-length scenario [19].

Many authors have studied effects of a minimal length in cosmology using a classical approach in which the Poisson brackets are modified according to the correspondence principle [10,22,47-51]. In the vast majority of the GUP formulation the parameter related to the minimal length (deformation parameter) is positive. Although GUP with positive deformation parameter prevents black holes to evaporate completely, at the same time they remove the Chandrasekhar limit, ${ }^{3}$ that is, a white dwarf star could turn out arbitrarily large [52,53]. In $[18,20]$ the author shows that GUP with a negative deformation parameter can restore the Chandrasekhar limit and, in spite of allowing a black hole to evaporate completely, this evaporation takes an infinite amount of time. Even though a negative deformation parameter is unusual, it is consistent with a description in which the universe has an underlying crystal lattice-like structure [54].

Mu-In Park proposed that the HUP can be modified to include the cosmology constant term, which is called extend uncertainty principle (EUP) $[35,55]$. Whereas GUP has a quadratic term in the momentum uncertainty, EUP has a quadratic term in the position uncertainty. GUP that has also a quadratic term in the position uncertainty is called GEUP. In [4] the authors use GEUP to show that a non-zero cosmological constant can restore the Chandrasekhar limit. Effects of GEUP in cosmology has been studied in [56]. Modified uncertainty principles that induce a maximum length have been employed in order to describe the cosmological particle horizon [57]. This modified uncertainty principle has terms proportional to even powers in the position uncertainty [58]. Cosmological observational data have been used to obtain constraints for GUP parameters [21,42,48,56,59].

\footnotetext{
2 The 3rd quantization consists of initially considering the WheelerDeWitt equation as a classical equation of a classical field (the wave function of the Universe in the 2nd quantization) which then is turned an operator. In this way universes can be created and annihilated in a multiverses theory.

3 The maximum mass of a stable white dwarf star.
}

Even though the de Sitter Cosmology Model describes the rapidly expansion phase of the Universe, during which vacuum energy dominates, its traditional treatment considers a system without physical content since there is only a single degree of freedom and one constraint [60]. Schutz formalism [61,62], which describes a relativistic fluid interacting with the gravity field, can be used to regard the vacuum as a dynamic entity having different degrees of freedom. This procedure overcomes the difficulties found in de Sitter Model and it leads to a natural way of introducing a variable playing the role of time. Then, the Dynamical Vacuum Model can be implemented in a homogeneous and isotropic universe filled with vacuum fluid whose state equation is $P=-\rho$, which is classically treated according to the Schutz canonical formalism. This process implies a linear term in one of the momenta in the Hamiltonian whose associated degree of freedom will play the role of time [63].

In general, with the intention of avoiding the problems caused by the WDW equation defined in the superspace ${ }^{4}$ (the space of all possible three dimensional metrics), the process of quantization is performed using the mini-superspace approach [64], where an infinite number of degrees of freedom of the gravity field is frozen ${ }^{5}$ and remaining degrees of freedom are turned operators.

Of course, if GUP is a fundamental aspect of the nature then not only the position and the momentum have to obey the GUP but also every other variables which will be quantized [65]. Since GUP corresponds to a modification of the commutation relation between the operator and its conjugate operator, the WDW equation in a minimal-length scenario can be obtained imposing that some or all of those operators (which have come from the quantization of remaining degrees of freedom in the mini-superspace approach) and its conjugate momentum operators satisfy modified commutation relations.

In this work, our primary purpose is to determine the corrections in the wave function of the Universe due to the use of a specific generalized uncertainty principle (GUP) in a quantum cosmology model. Hence, we determine the modified WDW equation up to $\mathcal{O}\left(\beta^{2}\right)$ considering a quantum cosmology model of dynamical vacuum in a minimal-length scenario induced by the commutation relation proposed by Pedram [66,67],

$[\hat{X}, \hat{P}]:=\frac{i \hbar}{1-\beta \hat{P}^{2}}$,

\footnotetext{
${ }^{4}$ As far as we know, there is no known general solution for the WDW equation in the superspace.

5 In fact, the mini-superspace approach is an approximation in which are only considered the largest gravity field wavelength modes of the order of the size of the Universe.
} 
which induces a minimal uncertainty in the position, $\Delta x_{\min }=$ $\frac{3 \sqrt{3}}{4} \hbar \sqrt{\beta}$, and an upper bound on the conjugate momentum, $P_{\text {max }}=\frac{1}{\sqrt{\beta}}$. In the equation above $\beta$ is a parameter related to the minimal length. An important aspect of the GUP associated with this modified commutation relation is that is not perturbative (in the minimal length). Moreover, it is consistent with Doubly Special Relativity (DSR) theories which predicts an upper bound for particle momentum [59,68-70] and it is in agreement with several theoretical proposals for quantum gravity [66]. We find the wave function of the Universe for small scale factors in the formal representation of the "position" space, in fact representation of the scale-factor space. ${ }^{6}$ However, it is not possible to obtain from wave function any physical information since the eigenstates of the "position" operators are not physical states [37]. We overcome this problem by obtaining the wave function in the representation of the quasi-position space as a superposition of wave functions in the formal representation of the "position" space [71]. Even if it may be questioned whether this procedure is correct, we find the modified WDW equation in the representation of the quasi-position space and we show that the wave function in the quasi-position space previously obtained is solution this equation, as we expected. Although the wave functions in the quasi-position space, which are eigenfunctions of the cosmological constant, are physically acceptable states they are not realizable states of the Universe because have infinite norm. Consequently, it will be necessary to construct wave packets from the superposition of eigenfunctions with different eigenvalues of the cosmological constant. Last but not least, it should be said that the quantization process occurs into the mini-superspace scenario in which the scale factor is the only degree of freedom. As far as we know, the first applications of a minimal-length scenario to a mini-superspace dynamics can be found in $[5,7,8]$.

In short, in this paper we intend to study a dynamical vacuum, using a description given by the Schutz formalism, in a quantum cosmological scenario where the minimal-length proposal is explicitly considered. Our main aim is to verify how GUP affects the solutions for the WDW equation with a dynamical vacuum state, in the mini-superspace, in comparison with the usual approach.

The paper is organized in the following way. In Sect. 2, we describe the minimal-length scenario used, presenting its main results. In Sect. 3, we describe the ordinary cosmology model, that is, in a scenario without minimal length. We obtain the gravity action and the fluid action according to the Schutz formalism. In Sect. 4, we determine the modified WDW equation in the formal representation of the "position" space and we find its solution for small values of the scale

\footnotetext{
${ }^{6}$ We say representation of the "position" space keeping in mind that it should be the more correct to call representation of the scale-factor space.
}

factor. We also discuss about the validity range for which the approach employed here is consistent. Then, we find the physically acceptable solutions, that is, the wave function of the Universe in the representation of the quasi-position space. Last we show that the norm of that wave function is infinite, as in the ordinary case with no minimal length. In Sect. 5, we present our comments and conclusions.

\section{Minimal-length scenario}

In a quantum approach, a minimal length can be introduced by modifying the HUP in order to implement a non-zero minimal uncertainty in the position. ${ }^{7}$ There are many proposals for modification of HUP [14,37, 38, 50, 57, 66, 67, 73].

In this work we concern with the GUP

$$
\Delta X \Delta P \geq \frac{\frac{\hbar}{2}}{1-\beta\left[(\Delta P)^{2}+\langle\hat{P}\rangle^{2}\right]},
$$

proposed by Pedram [66,67], which induces a non-zero minimal uncertainty in the position given by

$\Delta x_{\min }=\frac{3 \sqrt{3}}{4} \hbar \sqrt{\beta}$,

where $\beta$ is a parameter related to the minimal length. We choose the GUP (1) because it is consistent with several proposals for quantum gravity, such as string theory, loop quantum gravity, and it also introduces a maximal measured momentum, ${ }^{8}$

$P_{\max }=\frac{1}{\sqrt{\beta}}$,

which is in agreement with the DSR. Another important aspect this GUP is that it is not perturbative. Consequently, if $\beta$ is small then (1) can be expanded until any order in $\beta$ we wish.

Since,

$\Delta X \Delta P \geq \frac{1}{2}|\langle[\hat{X}, \hat{P}]\rangle|$

then related to the GUP (1) we have the modified commutation relation,

$[\hat{X}, \hat{P}]:=\frac{i \hbar}{1-\beta \hat{P}^{2}}$.

Although, the representation of the operators:

$\hat{X}=\hat{x}$

\footnotetext{
$\overline{7}$ It is not trivial to show that a non-zero minimal uncertainty in position can be interpreted as a minimal length $[23,37,72]$.

${ }^{8}$ It is worth noting that this GUP does not induce a maximum uncertainty in the conjugate momentum.
} 
$\hat{P}=\hat{p}+\frac{\beta}{3} \hat{p}^{3}+\frac{\beta^{2}}{3} \hat{p}^{5}+\frac{4 \beta^{3}}{9} \hat{p}^{7}+\cdots$,

where $\hat{x}$ and $\hat{p}$ are the ordinary operators of position and momentum satisfying the canonical commutation relation $[\hat{x}, \hat{p}]:=i \hbar$, is not an exact representation of the algebra (5), it preserves the ordinary form of the position operator. Thus, in this representation of "position" space we have,

$$
\begin{aligned}
& \langle x|\hat{X}| \psi(t)\rangle=x \psi(x, t) \\
& \langle x|\hat{P}| \psi(t)\rangle \\
& =\left(-i \hbar \frac{\partial}{\partial x}+i \hbar^{3} \frac{\beta}{3} \frac{\partial^{3}}{\partial x^{3}}-i \hbar^{5} \frac{\beta^{2}}{3} \frac{\partial^{5}}{\partial x^{5}}+\ldots\right) \psi(x, t)
\end{aligned}
$$

where $|x\rangle$ are the state eigenvectors of the position operator.

This representation of "position" space is only formal since the eigenstates of the position operator (6) are not physical states and consequently they do not belong to the Hilbert space. This is because the position operator uncertainty vanishes when it is calculated in any of its eigenstates. But that is physically impossible since $\Delta x \geq \frac{3 \sqrt{3}}{4} \hbar \sqrt{\beta}$ for all physically allowable state in a minimal-length scenario. However, all information on position can be accessible through the maximal localization states, defined as,

$\left\langle\psi_{\xi}^{m l}|\hat{X}| \psi_{\xi}^{m l}\right\rangle=\xi$

and

$$
(\Delta x)_{\left|\psi_{\xi}^{m l}\right\rangle}=(\Delta x)_{\min } .
$$

In the DGS (Detournay, Gabriel and Spindel) approach [74] the maximal localization states are found to be, ${ }^{9}$

$\psi_{\xi}^{m l}(p)=\sqrt{\frac{2 \sqrt{3}}{2}} \exp \left(-\frac{i}{\hbar} \xi p\right) \cos \left(\frac{3 \pi}{4} \sqrt{\beta} p\right)$.

The representation of quasi-position space is obtained by projecting the state vectors onto the maximal localization states,

$\left\langle\psi_{\xi}^{m l} \mid \psi(t)\right\rangle=\psi_{q p}(\xi, t)$.

The action of the position and the momentum operators on the quasi-position space are given by,

$\left\langle\psi_{\xi}^{m l}|\hat{X}| \psi(t)\right\rangle=\left[\xi+i b \sqrt{\beta} \tan \left(-i b \sqrt{\beta} \frac{\partial}{\partial \xi}\right)\right] \psi_{q p}(\xi, t)$

and

$\left\langle\psi_{\xi}^{m l}|\hat{P}| \psi(t)\right\rangle=\left\langle\psi_{\xi}^{m l}\left|\left(\hat{p}+\frac{\beta}{3} \hat{p}^{3}+\frac{\beta^{2}}{3} \hat{p}^{5}+\ldots\right)\right| \psi(t)\right\rangle$,

\footnotetext{
${ }^{9}$ Note that it is in the representation of the ordinary momentum space.
}

with

$\left\langle\psi_{\xi}^{m l}|\hat{p}| \psi(t)\right\rangle=-i \hbar \frac{\partial \psi_{q p}(\xi, t)}{\partial \xi}$,

and

$b:=\frac{3 \pi \hbar}{4}$.

Hence, up to $\mathcal{O}\left(\beta^{2}\right)$ we have

$$
\left\langle\psi_{\xi}^{m l}|\hat{X}| \psi(t)\right\rangle=\left(\xi+\beta b^{2} \frac{\partial}{\partial \xi}-\beta^{2} \frac{b^{4}}{3} \frac{\partial^{3}}{\partial \xi^{3}}\right) \psi_{q p}(\xi, t),
$$

and

$$
\begin{aligned}
& \left\langle\psi_{\xi}^{m l}|\hat{P}| \psi(t)\right\rangle \\
& =\left(-i \hbar \frac{\partial}{\partial \xi}+i \hbar^{3} \frac{\beta}{3} \frac{\partial^{3}}{\partial \xi^{3}}-i \hbar^{5} \frac{\beta^{2}}{3} \frac{\partial^{5}}{\partial \xi^{5}}\right) \psi_{q p}(\xi, t) .
\end{aligned}
$$

Finally, one can show that the wave function in the quasiposition space is a superposition of the wave functions in the formal "position" space, given by

$\psi_{q p}(\xi)=\frac{1}{\sqrt{2}}[\psi(\xi+b \sqrt{\beta})+\psi(\xi-b \sqrt{\beta})]$.

\section{The model}

We will now consider the de Sitter Cosmology Model, which describes the phase of the Universe with an exponential expansion, during which the vacuum energy dominates the energy density and gives rise the term corresponding to the cosmological constant $\Lambda$.

\subsection{Gravity action}

The more general gravity action leading up to second order differential equations is given by, ${ }^{10}$

$S_{g}=\int_{M} d^{4} x \sqrt{-g}(R-2 \Lambda)+2 \int_{\partial M} d^{3} x \sqrt{h} K$,

where $R$ is the Ricci curvature scalar, $K$ is the trace of the extrinsic curvature $K_{i j}, g$ is the determinant of the metric $g_{\mu \nu}, h$ is the determinant of the induced metric over the threedimensional spatial hypersurface and $\partial M$ is the bounded of the four-dimensional manifold $M$. The cosmological constant $\Lambda$ appears as a geometric term and its value is arbitrary. In fact, the first integral in the 1.h.s. of (21) constitutes the most general geometrical Lagrangian in four dimensions leading to second order differential equations according to the Lovelock theorem [75].

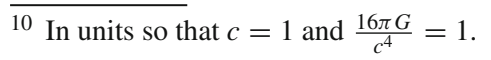


According to the Cosmological Principle, that is, for a homogeneous and isotropic universe, the metric is that of FLRW (Friedmann-Lemaître-Robertson-Walker),

$$
\begin{aligned}
d s^{2}= & -N^{2}(t) d t^{2} \\
& +a^{2}(t)\left[\frac{d r^{2}}{\left(1-k r^{2}\right)}+r^{2} d \theta^{2}+r^{2} \sin ^{2} \theta d \phi^{2}\right],
\end{aligned}
$$

where $N(t)$ is the lapse function, $k=-1,0,+1$ for hyperbolic open, flat open and close universe, respectively, and $a(t)$ is the scale factor of universe.

Using the FLRW metric, Eq. (22), the gravity action becomes

$S_{g}=\int d t\left(-\frac{6 \dot{a}^{2} a}{N}+k N a-2 N a^{3} \Lambda\right)$.

Since $S_{g}=\int d t L_{g}$ we have that the gravity Lagrangian is given by

$L_{g}=-\frac{6 \dot{a}^{2} a}{N}+k N a-2 N a^{3} \Lambda$.

Hence the gravity Hamiltonian, $N H_{g}=\dot{a} p_{a}-L_{g}$, reads

$H_{g}=-\frac{p_{a}^{2}}{24 a}-k a+2 \Lambda a^{3}$,

where

$p_{a}=\frac{\partial L_{g}}{\partial \dot{a}}=-\frac{12 \dot{a} a}{N}$.

\subsection{Fluid action}

In our model the Universe is fully composed of perfect fluid. Now, we will employ the Schutz's formalism [61,62] for the dynamics description the fluid interacting with the gravitational field [63]. For the fluid which the state equation is,

$p=\alpha \rho$,

where $\rho$ is the density and $\alpha$ is the equation of state parameter depending on the type of fluid, the pressure is given by,

$p=\alpha\left(\frac{\mu}{\alpha+1}\right)^{1+\frac{1}{\alpha}} e^{-\frac{S}{\alpha}}$,

where $\mu$ is the specific enthalpy and $S$ is the entropy.

In the Schutz's formalism the four-velocity is defined by means of six potentials:

$U_{v}:=\frac{1}{\mu}\left(\phi,{ }_{\nu}+\epsilon \eta,{ }_{\nu}+\theta S,{ }_{\nu}\right)$.

Since $\epsilon$ and $\eta$ are associated with rotation movement, they vanish. From the normalization condition,

$U^{v} U_{v}=-1$, we obtain,

$\mu=\frac{1}{N}(\dot{\phi}+\theta \dot{S})$.

The fluid action is given by

$S_{F}=\int_{M} d^{4} x \sqrt{-g} p$.

Using Eq. (31) into Eq. (28) and since the spatial section is homogeneous and isotropic, we have,

$S_{F}=\int d t N a^{3} \alpha\left[\frac{\dot{\phi}+\theta \dot{S}}{N(\alpha+1)}\right]^{1+\frac{1}{\alpha}} e^{-\frac{S}{\alpha}}$.

Thus, the fluid Lagrangian is,

$L_{F}=\frac{\alpha a^{3}}{N^{\frac{1}{\alpha}}(1+\alpha)^{1+\frac{1}{\alpha}}}(\dot{\phi}+\theta \dot{S})^{1+\frac{1}{\alpha}} e^{-\frac{S}{\alpha}}$.

The conjugate canonical momenta to $\phi$ and $S$ are,

$p_{\phi}=\frac{\partial L_{F}}{\partial \dot{\phi}}=\frac{a^{3}(\dot{\phi}+\theta \dot{S})^{\frac{1}{\alpha}} e^{-\frac{S}{\alpha}}}{N^{\frac{1}{\alpha}}(1+\alpha)^{\frac{1}{\alpha}}}$

and

$p_{S}=\frac{\partial L_{F}}{\partial \dot{S}}=\theta p_{\phi}$.

Therefore, the fluid Hamiltonian, $N H_{F}=\dot{\phi} p_{\phi}+\dot{S} p_{S}-$ $L_{F}$, is given by,

$H_{F}=\frac{p_{\phi}^{1+\alpha} e^{S}}{a^{3 \alpha}}$.

From Eqs. (36) and (25), it follows that the total Hamiltonian, $H=H_{g}+H_{F}$, is

$H=-\frac{p_{a}^{2}}{24 a}-k a+2 \Lambda a^{3}+\frac{p_{\phi}^{1+\alpha} e^{S}}{a^{3 \alpha}}$.

Now, performing the canonical transformation (re-parametrization)

$T:=-p_{S} e^{-S} p_{\phi}^{-(1+\alpha)}$,

and

$p_{T}:=p_{\phi}^{1+\alpha} e^{S}$,

the total Hamiltonian becomes,

$H=-\frac{p_{a}^{2}}{24 a}-k a+2 a^{3} \Lambda+\frac{p_{T}}{a^{3 \alpha}}$.

Note that $p_{T}$ is linear in Eq. (41). When the quantization is implemented this fact allows us to consider $t=-T$ as playing role of time and to obtain a Schrödinger-like equation. 


\section{The quantum cosmology model in a minimal-length scenario}

\subsection{The modified Wheeler-DeWitt equation}

The quantization process takes place in the framework of mini-superspace and according with the Wheeler-DeWitt quantization scheme,

$p_{a} \longrightarrow \hat{p}_{a}$

and

$p_{T} \longrightarrow \hat{p}_{T} \equiv-i \frac{\partial}{\partial T}$,

such that $H \longrightarrow \hat{H}$, implying the WDW equation,

$\hat{H}|\psi\rangle=0$.

In order to obtain a minimal-length scenario we demand that

$\left[\hat{a}, \hat{p}_{a}\right]:=\frac{i \hbar}{1-\beta \hat{p}_{a}^{2}}$.

with the representation of the operators as follow:

$\hat{a}=\hat{x}$

and

$\hat{p}_{a} \equiv \hat{p}+\frac{\beta}{3} \hat{p}^{3}+\frac{\beta^{2}}{3} \hat{p}^{5}+\mathcal{O}\left(\beta^{3}\right)$,

where the $\hat{x}$ and $\hat{p}$ operators satisfy the canonical commutation relation,

$[\hat{x}, \hat{p}]:=i \hbar$.

Using the representation (46) and (47), the modified WDW equation turns out

$$
\begin{aligned}
- & \frac{\hat{x}^{(3 \alpha-1)}}{24}\left[\hat{p}^{2}+\frac{2}{3} \beta \hat{p}^{4}+\frac{7}{9} \beta^{2} \hat{p}^{6}+\mathcal{O}\left(\beta^{3}\right)\right]|\psi(t)\rangle \\
& +\left[-k \hat{x}^{(1+3 \alpha)}+2 \Lambda \hat{x}^{3(1+\alpha)}\right]|\psi(t)\rangle=-i \frac{\partial}{\partial t}|\psi(t)\rangle,
\end{aligned}
$$

where we have made $T=-t$.

Now, projecting Eq. (50) onto the formal representation of the "position" space we have,

$$
\begin{aligned}
& \langle x|\hat{H}| \psi(t)\rangle=0, \\
& -\frac{x^{(3 \alpha-1)}}{24}\left[-\frac{\partial^{2}}{\partial x^{2}}+\frac{2}{3} \beta \frac{\partial^{4}}{\partial x^{4}}-\frac{7}{9} \beta^{2} \frac{\partial^{6}}{\partial x^{6}}+\mathcal{O}\left(\beta^{3}\right)\right] \psi(x, t) \\
& +\left[-k x^{(1+3 \alpha)}+2 \Lambda x^{3(1+\alpha)}\right] \psi(x, t)=-i \frac{\partial}{\partial t} \psi(x, t),
\end{aligned}
$$

where we have used Eqs. (8) and (9).

Assuming that the solution of the above equation can been written as $\psi(x, t)=\varphi(x) \tau(t)$, we find the stationary solutions,

$\psi_{\omega}(x, t)=e^{-i \omega t} \varphi_{\omega}(x)$, and the time independent equation,

$$
\begin{aligned}
& {\left[\frac{d^{2}}{d x^{2}}-\frac{2}{3} \beta \frac{d^{4}}{d x^{4}}+\frac{7}{9} \beta^{2} \frac{d^{6}}{d x^{6}}+\mathcal{O}\left(\beta^{3}\right)\right] \varphi_{\omega}(x)} \\
& +\left[24 x^{(1-3 \alpha)} \omega-24 k x^{2}+48 \Lambda x^{4}\right] \varphi_{\omega}(x)=0,
\end{aligned}
$$

where $\omega$ is a constant.

The equation of state parameter $\alpha$ defines the matter content in a single fluid model. The case $\alpha=-1$ corresponds to the vacuum energy equation of state. It comes from the quantum field contributions for the energy associated with the vacuum state. Its precise value is the object of discussions today, and it can be related to the dark energy component in the Universe. Here, we consider it as a dynamic fluid component with internal degrees of freedom. Remark that the contribution of this vacuum component for the matter content, given by $\alpha=-1$, is similar to the geometric cosmological constant $\Lambda$, but the physical meaning and origin are very different.

Lastly, considering a flat universe ${ }^{11}(k=0)$, without any other matter content, fully completed by a dynamical vacuum with an equation of state,

$P=-\rho$,

and $\Lambda=0,{ }^{12}$ Eq. (53) up to $\mathcal{O}\left(\beta^{2}\right)$ becomes, ${ }^{13}$

$\frac{d^{2} \varphi}{d x^{2}}-\frac{2}{3} \beta \frac{d^{4} \varphi}{d x^{4}}+\frac{7}{9} \beta^{2} \frac{d^{6} \varphi}{d x^{6}}+24 \omega x^{4} \varphi=0$.

\subsection{Solution of the modified Wheeler-DeWitt equation}

It is not possible to solve Eq. (55) analytically since we do not know all initial or boundary conditions, ${ }^{14}$ that is, $\varphi^{(N)}\left(x_{0}\right)$, for $N=0,1, \ldots, 5$. A way to get around this problem is considering $x$ small. This is well reasonable since we are interested in solutions describing the Universe in its quantum regime, that is, in the initial phases of the Universe, when the scale factor was small ${ }^{15}$ and as it is known quantum effects are significant only for small values of the scale factor.

\footnotetext{
11 We choose a flat universe because it is in agreement with current cosmological observations [76,77].

12 In the same way as in [63] we hope that the cosmological constant arises from the degrees of freedom of the vacuum and not being postulated from the start as a geometric term. Note also that if from the start $\Lambda>0$ we can always redefine $\omega$ as $\omega /:=\omega+2 \Lambda$.

${ }^{13}$ From now on, for the sake of simplicity we are going to omit the subscript $\omega$.

${ }^{14}$ In Ref. [2] the author has solved a modified WDW equation of forth order $\left(\frac{d^{4}}{d x^{4}}\right)$ using the Sommerfeld polynomial method. However, the solution holds dependent on four parameters that must be determined by the initial conditions, which are not known.

15 Later on, we will discuss more carefully the issue if $x$ can rigorously describe the scale factor.
} 
Initially, we take into account Eq. (55) in the limit $\beta=0$, which we call ordinary WDW equation,

$\frac{d^{2} \varphi_{0}}{d x^{2}}+24 \omega x^{4} \varphi_{0}=0$

Making $\varphi_{0}=\sqrt{x} y(x)$ and the change of variable $\rho:=$ $\sqrt{\frac{8 \omega}{3}} x^{3}$, Eq. (55) turns the Bessel equation,

$\frac{d^{2} y}{d \rho^{2}}+\frac{1}{\rho} \frac{d y}{d \rho}+\left[1-\frac{\left(\frac{1}{6}\right)^{2}}{\rho^{2}}\right] y=0$.

Then, the solution of Eq. (57) is

$\varphi_{0}(x)=A \sqrt{x} J_{1 / 6}\left(K_{0} x^{3}\right)+B \sqrt{x} N_{1 / 6}\left(K_{0} x^{3}\right)$,

where $K_{0}:=\sqrt{\frac{8 \omega}{3}}, J_{1 / 6}(X)$ and $N_{1 / 6}(X)$ are the Bessel and the Neumann functions of order $1 / 6$, respectively, and $A$ and $B$ are constants.

It is easy to see that in the limit for small $x$,

$J_{1 / 6}(X) \approx \sqrt{x}$

and

$N_{1 / 6}(X) \approx \frac{1}{\sqrt{x}}$.

Now, imposing the condition ${ }^{16} \varphi_{0}(0)=0$, we get $B=0$, and the solution is given by,

$\varphi_{0}(x)=A \sqrt{x} J_{1 / 6}\left(K_{0} x^{3}\right)$.

Remembering that

$J_{v}(X)=\sum_{N=0}^{\infty} \frac{(-1)^{N}}{N ! \Gamma(N+v+1)}\left(\frac{X}{2}\right)^{2 N+v}$,

and retaining the first three significant terms of $\varphi_{0}(x)$ we get,

$\varphi_{0}(x)=C_{1} x+C_{2} x^{7}+C_{3} x^{13}$.

It is appropriate to rewrite the solution (63) by absorbing $C_{1}$ in a normalization constant, which we are going to omit without affecting the results:

$\varphi_{0}(x)=x-\frac{4}{7} \omega x^{7}+\frac{8}{91} \omega^{2} x^{13}$.

We can obtain an approximate solution for Eq. (55), in the range of $x$ small, using Eq. (64) into $\mathcal{O}(\beta)$ and $\mathcal{O}\left(\beta^{2}\right)$ terms. Thus,

$\frac{d^{4} \varphi_{0}}{d x^{4}}=-480 \omega x^{3}+\frac{10560}{7} \omega^{2} x^{9}$

$\overline{16}$ That condition is obtained by demanding that the Hamiltonian $H$ must be self-adjoint [63]. That condition should not change, even though it is probably that boundary conditions satisfied by derivatives change. Note that condition does not avoid the initial singularity $[8,9]$. and

$\frac{d^{6} \varphi_{0}}{d x^{6}}=-2880 \omega x+\frac{760320}{7} \omega^{2} x^{7}$.

With the approximation $\varphi \approx x$ into Eqs. (65) and (66), Eq. (55) becomes,

$$
\begin{aligned}
& \frac{d^{2} \varphi}{d x^{2}}+24 \omega x^{4} \varphi+\beta\left(320 \omega x^{2} \varphi-\frac{7040}{7} \omega^{2} x^{8} \varphi\right) \\
& +\beta^{2}\left(-2240 \omega \varphi+591360 \omega^{2} x^{6} \varphi\right)=0 .
\end{aligned}
$$

Subsequently, we are going to find the approximate solution of the above equation in two different ways.

\subsubsection{Solution: first method}

Since $x=0$ is an ordinary point of Eq. (67) we can find a solution in power series of $x$. Then, replacing $\varphi(x)=$ $\sum_{n=0}^{\infty} a_{n} x^{n}$ into Eq. (67) and retaining only significant terms, we have,

$$
\begin{aligned}
& \varphi(x)=x-\frac{4}{7} \omega x^{7}+\frac{8}{91} \omega^{2} x^{13} \\
& +\beta\left(-16 A \omega x^{5}+\frac{5504}{385} B \omega^{2} x^{11}\right)+\beta^{2} \frac{1120}{3} C \omega x^{3} .
\end{aligned}
$$

The parameters $A, B$ and $C$ are determined by requiring that (68) satisfies Eq. (67) up to order chosen in $x$. Then, substituting (68) into (67) we have $A=1, B=\frac{38}{43}$ and $C=\frac{3}{7}$. Therefore, the solution reads as,

$$
\begin{aligned}
& \varphi(x)=x-\frac{4}{7} \omega x^{7}+\frac{8}{91} \omega^{2} x^{13} \\
& +\beta\left(-16 \omega x^{5}+\frac{4864}{385} \omega^{2} x^{11}\right)+\beta^{2} 160 \omega x^{3} .
\end{aligned}
$$

\subsubsection{Solution: second method}

The same previous solution can be obtained by taking the approximation following,

$\frac{d^{2} \varphi}{d x^{2}}=-24 \omega x^{4} \varphi_{0}+\frac{2}{3} \beta \frac{d^{4} \varphi_{0}}{d x^{4}}-\frac{7}{9} \beta^{2} \frac{d^{6} \varphi_{0}}{d x^{6}}$.

Integrating twice the above equation we obtain,

$$
\begin{aligned}
& \frac{d^{2} \varphi}{d x^{2}}=-24 \omega \int d x \int d x x^{4} \varphi_{0} \\
& +\frac{2}{3} \beta \frac{d^{2} \varphi_{0}}{d x^{2}}-\frac{7}{9} \beta^{2} \frac{d^{4} \varphi_{0}}{d x^{4}}+\alpha_{1} x+\alpha_{2},
\end{aligned}
$$

where $\alpha_{1}$ and $\alpha_{2}$ are integration constants. After that, using (64) into (71) we obtain,

$$
\varphi(x)=\alpha_{2}+\alpha_{1} x-\frac{4}{7} \omega x^{7}+\frac{8}{91} \omega^{2} x^{13}
$$




$$
+\beta\left(-16 A^{\prime} \omega x^{5}+\frac{64}{7} B^{\prime} \omega^{2} x^{11}\right)+\beta^{2} \frac{120}{3} C^{\prime} \omega x^{3} .
$$

The constants $\alpha_{1}$ and $\alpha_{2}$ can be determined by demanding that $\varphi(x) \longrightarrow \varphi_{0}(x)$ when $\beta \longrightarrow 0$. In a similar way, the parameters $A^{\prime}, B^{\prime}$ and $C^{\prime}$ are found requiring that the solution (72) satisfies Eq. (67). Consequently, we again have the solution (69), as we expected.

\subsection{Validity range}

In order that the solution (69) works consistently the first disregarded term in $\varphi_{0}(x)$ must be much smaller than the last kept terms in $\mathcal{O}(\beta)$ and $\mathcal{O}\left(\beta^{2}\right)$, that is, $x \ll\left(\frac{\beta}{\omega}\right)^{\frac{1}{8}}$. Since $\beta$ is much small, this drastically reduces the validity range for our solution. We can improve this if terms of higher powers in $x$ are taken account in order $\mathcal{O}\left(\beta^{0}\right)$ part of the solution. Therefore we can consider $\sqrt{x} J_{1 / 6}\left(K_{0} x^{3}\right)$ as the part in $\mathcal{O}\left(\beta^{0}\right)$ of $\varphi(x)$, that is, $\varphi_{0}(x)$. It is clear that terms of order such that $x^{6 n-2}<\beta^{2} \omega^{1-n}$ lie far outside the validity range and they must throw away.

The same reasoning applies in the case of terms in $\mathcal{O}\left(\beta^{2}\right)$ when compared to terms in $\mathcal{O}(\beta)$. This way, with the purpose of increasing the validity range of our solution we take account the two first significant terms in $\mathcal{O}(\beta)$. In conclusion, in light of the foregoing the solution turns out to be, ${ }^{17}$

$$
\begin{aligned}
& \varphi(x)=\sqrt{x} J_{1 / 6}\left(K_{0} x^{3}\right) \\
& +\beta\left(-16 \omega x^{5}+\frac{4864}{385} \omega^{2} x^{11}\right)+\beta^{2} 160 \omega x^{3} .
\end{aligned}
$$

In dealing with expansions of small parameters (in this case $\beta$ ) it is necessary to take care to consistently work when $x$ goes to an extremely small value. It is easy to see that terms in $\beta^{0}$ are like $\omega^{N} x^{1+6 N}$, terms in $\beta$ are like $\beta \omega^{N} x^{5+6 N}$ and terms in $\beta^{2}$ are like $\beta^{2} \omega^{N} x^{3+6 N}$. Therefore, the terms in $\beta \omega x^{5}$ and $\beta \omega^{2} x^{11}$ are negligible when $x<\sqrt{\beta}$ and the term $\beta \omega^{2} x^{11}$ is negligible when $\sqrt{\beta}<x<\beta^{1 / 8}$. Note that the term in $\beta^{0} x$ always will be greater than the term in $\beta^{2} \omega x^{3}$ for $x$ small.

Keep in mind that in fact solution (73) is $\varphi_{\omega}(x)$, that is, eigenfunctions whose associated eigenvalues are values of the cosmological constant.

\subsection{Physically acceptable solutions}

As we have already said, we can not obtain directly from $\varphi(x)$ physical results because the $\hat{x}$ operator eigenstates do not belong to the Hilbert space. Nevertheless, the projections of the state vectors $|\varphi\rangle$ on the maximal localization states, that is, the wave functions in the representation of the quasiposition space do. The wave functions in the quasi-position

\footnotetext{
17 Note that solution is non-normalized.
}

space can easily be obtained from (73) by using Eq. (20). Therefore, replacing (73) into (20) we have ${ }^{18}$

$$
\begin{aligned}
& \varphi_{q p}(\xi)=\sqrt{\xi} J_{1 / 6}\left(K_{0} \xi^{3}\right) \\
& +\beta\left[-\left(16+12 b^{2}\right) \omega \xi^{5}+\left(\frac{4864}{385}+\frac{48}{7} b^{2}\right) \omega^{2} \xi^{11}\right] \\
& +\beta^{2}\left(160-160 b^{2}-20 b^{4}\right) \omega \xi^{3}
\end{aligned}
$$

Hence, above quasi-position wave function represents the probability amplitude for the Universe being maximally localized around the position $\xi$.

The reader may be questioning if the correct procedure would be to determine the solution of the modified WDW equation in the representation of the quasi-position space,

$\left\langle\psi_{\xi}^{m l}\left|\left(-\hat{p}_{a}^{2}+24 \omega \hat{a}^{4}\right)\right| \psi(t)\right\rangle=0$.

Using Eqs. (18) and (19) we obtain

$$
\begin{aligned}
& \frac{d^{2} \varphi_{q p}}{d \xi^{2}}+24 \omega \xi^{4} \varphi_{q p} \\
& +\beta b^{2}\left[-\frac{2}{3 b^{2}} \frac{d^{4} \varphi_{q p}}{d \xi^{4}}+144 \omega \xi^{2} \varphi_{q p}+96 \omega \xi^{3} \frac{d \varphi_{q p}}{d \xi}\right] \\
& +\beta^{2} b^{4}\left[-\frac{7}{9 b^{4}} \frac{d^{6} \varphi_{q p}}{d \xi^{6}}+24 \omega \varphi_{q p}+96 \omega \xi \frac{d \varphi_{q p}}{d \xi}\right] \\
& +\beta^{2} b^{4}\left[-24 \omega \xi^{2} \frac{d^{2} \varphi_{q p}}{d \xi^{2}}-40 \omega \xi^{3} \frac{d^{3} \varphi_{q p}}{d \xi^{3}}\right]=0 .
\end{aligned}
$$

As it can easily be checked (after some algebra) the solution (74) satisfies Eq. (76) up to the considered validity order.

\subsection{Norm of the wave function}

In the ordinary case the norm of the wave function solution of the WDW equation is infinite [63]. This means that stationary states of the Universe with well-defined values of the cosmological constant are not realizable and it is necessary the construction of wave packets.

Now, we are going to calculate the norm of the wave function (74). Since the determination of the norm of the wave function in the quasi-position space can be laborious, it is convenient to perform first the calculation formally in the "position" space and after to obtain the normalized wave function (if it is normalized) in the quasi-position space using Eq. (20).

In the "position" space the inner product does not change, so

$$
\left\langle\psi_{1} \mid \psi_{2}\right\rangle=\int_{0}^{\infty} \psi_{2}^{*}(x) \psi_{1}(x) d x .
$$

\footnotetext{
${ }^{18}$ Remember that $b:=\frac{3 \pi}{4}$, Eq. (17).
} 
On the basis of solution (69) we assume that $\psi_{1}^{(n)}(0)=0$ for $n=0,2,4, \ldots$ Thus, it is easy to show that the operator,

$\hat{D}:=\frac{d^{2}}{d x^{2}}-\frac{2}{3} \beta \frac{d^{4}}{d x^{4}}+\frac{7}{9} \beta^{2} \frac{d^{6}}{d x^{6}}$

is self-adjoint. ${ }^{19}$

However, the operator $\hat{H}:=\hat{D}+24 \omega x^{4}$ is not self-adjoint in the inner product (77), but rather in the inner product

$$
\left\langle\psi_{1} \mid \psi_{2}\right\rangle=\int_{0}^{\infty} x^{4} \psi_{2}^{*}(x) \psi_{1}(x) d x
$$

where $x^{4}=w(x)$ is a weight function.

Some caution is necessary with the calculation of the norm of the solution (73) because the solution (73) is only valid for small values of $x$. Therefore it can not be used throughout the whole positive $x$-axis. Thus, we consider that the effects of the existence of a minimal length are negligible for values of $x$ greater than those of the validity range of the solution (73), say $x_{M}$. Consequently, the solution turns to the ordinary $\varphi_{0}(x)$ for $x>x_{M}$. In this way,

$$
\langle\varphi \mid \varphi\rangle=\int_{0}^{x_{M}} x^{4}|\varphi(x)|^{2} d x+\int_{x_{M}}^{\infty} x^{4}\left|\varphi_{0}(x)\right|^{2} d x .
$$

Note that if $x_{M} \approx\left(\frac{\beta}{\omega}\right)^{\frac{1}{8}}$ then $\varphi(x)$ of the first integral in the 1.h.s. of (80) is given by Eq. (69), according to Sect. 4.3. Because of the second integral in the 1.h.s. of (80) the norm is infinite. This result was already expected since the divergence takes place for $x \rightarrow \infty$ where the effects of a minimal length are negligible. In the ordinary case, the problem with the infinite norm is solved by constructing a wave packet. The construction of a wave packet when the minimal length is presented is much less simple due to the modifications given by the existence of a minimal length near the origin.

Although the wave function (74) can not be normalized, it is interesting to note that the leading correction term in $\mathcal{O}(\beta)$ of $\left|\varphi_{q p}(\xi)\right|^{2}$ is

$-2 \beta\left(16+12 b^{2}\right) \omega \xi^{6}$.

Consequently, $\left|\varphi_{q p}\right|^{2}$ grows more slowly than $\left|\varphi_{0}\right|^{2}$. In [47] the author ${ }^{20}$ has found that the leading correction term is positive implying a more rapid growth of $|\varphi|^{2}$.

$\overline{19}$ Note that $\hat{D}$ is not defined on the whole Hilbert space $\mathcal{H}$ because $\hat{D}$ is an unbounded linear operator. Thus, the domain of $\hat{D}$ is a dense subspace into $\mathcal{H}$.

20 The author has considered that the variable $u:=a^{\frac{2}{3}}$ and its conjugate momentum $p_{u}=\frac{2}{3} a^{-\frac{1}{2}} p_{a}$ satisfy the commutation relation proposed by Kempf, Mangano and Mann [37] when turned operators.

\section{Conclusion}

In this work, we performed the study of the primordial Universe by implementing a quantum approach in the gravitation effects. It follows the need to implement a minimal-length scenario, which is carried out by using a GUP. Since we chose the GUP (1) we can expand the representation of the momentum until $\mathcal{O}\left(\beta^{2}\right)$ and thus to obtain a modified WDW equation up to $\mathcal{O}\left(\beta^{2}\right)$, too.

We found the modified WDW equation in the formal representation of "position" space, Eq. (55), and its solution $\varphi(x)$, Eq. (73), because it is simpler than in the representation of quasi-position space. However, we can not obtain directly from $\varphi(x)$ physical results. Consequently we obtained the wave function of the Universe in the representation of quasiposition space, $\varphi_{q p}(\xi)$, as a superposition of two wave functions of the Universe in the formal representation of "position" space, $\varphi_{q p}(\xi)=\frac{1}{\sqrt{2}}\left[\varphi\left(\xi+x_{\min }\right)+\varphi\left(\xi-x_{\min }\right)\right]$. With the aim of insuring our result, we found the modified WDW equation in the representation of quasi-position space, Eq. (76), and we checked that $\varphi_{q p}(\xi)$ is actually its solution.

The ignorance of the initial or boundary conditions, that is, of the derivatives of the wave function does not allow us to find an exact solution for modified WDW equation, what forced us to seek a solution for small values of the scale factor of the Universe.

Since in the ordinary case the divergence in the norm of the wave function takes place for $x \rightarrow \infty$, the introduction of a minimal length in the theory can not change this, because its effects are only significant for small $x$. Therefore realizable states of the Universe are wave packets constructed by superposition of the stationary states of different values of $\omega$, that is, of the cosmological constant.

We should note that different changes in the WDW equation due to different GUP's have been found in the literature. For example, a linear GUP [38] has led to the emergence of third and fourth order derivatives in the modified WDW equation [2,11,12,39], whereas the KMM GUP [37] has only induced to fourth order derivatives $[5,19,47,78]$. Those results are in agreement with our modified WDW equation which also displays a term of fourth order derivative in $\mathcal{O}(\beta)$.

Fluid radiation is another model of interest to study since the radiation-dominated era has been presented in the early Universe when the quantum effects were relevant. For a radiation fluid $\alpha=\frac{1}{3}$ and the modified WDW equation is given by

$\frac{d^{2} \varphi}{d x^{2}}-\frac{2}{3} \beta \frac{d^{4} \varphi}{d x^{4}}+\frac{7}{9} \beta^{2} \frac{d^{6} \varphi}{d x^{6}}+24 \omega \varphi=0$.

At first sight we could easily obtain an analytical solution of the above equation. For this we suppose $\varphi(x)=e^{k x}$ in order to get an algebraic equation of 6 th order which can be transformed in one of 3rd order. However, we do not know 
the initial or boundary conditions in order to determine the 6 constants of the general solution. Again, we need to appeal to the approximation for small $x$.

In next works, we are going to study the effects on the scale factor evolution, applications of the obtained results and comparisons with others models and GUP's.

Acknowledgements We would like to thank FAPES, CAPES and $\mathrm{CNPq}$ (Brazil) for financial support.

Data Availability Statement This manuscript has no associated data or the data will not be deposited. [Authors' comment: This work is completely theoretical in nature and no experimental data has been listed.]

Open Access This article is licensed under a Creative Commons Attribution 4.0 International License, which permits use, sharing, adaptation, distribution and reproduction in any medium or format, as long as you give appropriate credit to the original author(s) and the source, provide a link to the Creative Commons licence, and indicate if changes were made. The images or other third party material in this article are included in the article's Creative Commons licence, unless indicated otherwise in a credit line to the material. If material is not included in the article's Creative Commons licence and your intended use is not permitted by statutory regulation or exceeds the permitted use, you will need to obtain permission directly from the copyright holder. To view a copy of this licence, visit http://creativecomm ons.org/licenses/by/4.0/.

Funded by SCOAP ${ }^{3}$.

\section{References}

1. S. Hossenfelder, A note on theories with a minimal length. Class. Quantum Gravity 23, 1815 (2006). https://doi.org/10.1088/ 0264-9381/23/5/N01

2. M. Kober, Generalized uncertainty principle in canonical quantum gravity and application to quantum cosmology. Int. J. Mod. Phys. A 27(20), 1250106 (2012). https://doi.org/10.1142/ S0217751X12501060

3. Y. Sabri, K. Nouicer, Phase transitions of a GUP-corrected Schwarzschild black hole within isothermal cavities. Class. Quantum Gravity 29(21), 215015 (2012). https://doi.org/10.1088/ $0264-9381 / 29 / 21 / 215015$

4. Y.C. Ong, Y. Yao, Generalized uncertainty principle and white dwarfs redux: how the cosmological constant protects the Chandrasekhar limit. Phys. Rev. D 98(12), 126018 (2018). https://doi. org/10.1103/PhysRevD.98.126018

5. B. Vakili, H.R. Sepangi, Generalized uncertainty principle in Bianchi type I quantum cosmology. Phys. Lett. B 651(2-3), 79 (2007). https://doi.org/10.1016/j.physletb.2007.06.015

6. M.V. Battisti, G. Montani, The big-bang singularity in the framework of a generalized uncertainty principle. Phys. Lett. B 656(1-3), 96 (2007). https://doi.org/10.1016/j.physletb.2007.09.012

7. M.V. Battisti, G. Montani, Minisuperspace dynamics in a generalized uncertainty principle framework, in AIP Conference Proceedings, vol. 966, p. 219 (2008). https://doi.org/10.1063/1.2836998

8. M.V. Battisti, G. Montani, Quantum dynamics of the Taub universe in a generalized uncertainty principle framework. Phys. Rev. D 77(2), 023518 (2008). https://doi.org/10.1103/PhysRevD.77. 023518
9. M.V. Battisti, G. Montani, Quantum cosmology with a minimal length. Int. J. Mod. Phys. A 23(8), 1257 (2008). https://doi.org/10. 1142/S0217751X08040184

10. A.F. Ali, B. Majumder, Towards a cosmology with minimal length and maximal energy. Class. Quantum Gravity 31(21), 215007 (2014). https://doi.org/10.1088/0264-9381/31/21/215007

11. M. Faizal, Deformation of the Wheeler DeWitt equation. Int. J. Mod. Phys. A 29(20), 1450106 (2014). https://doi.org/10.1142/ S0217751X14501061

12. R. Garattini, M. Faizal, Cosmological constant from a deformation of the Wheeler-DeWitt equation. Nucl. Phys. B 905, 313 (2016). https://doi.org/10.1016/j.nuclphysb.2016.02.023

13. O. Ökcü, C. Corda, E. Aydiner, Modified Friedmann equations from DSR-GUP. Europhys. Lett. 129(5), 50002 (2020). https://doi.org/ $10.1209 / 0295-5075 / 129 / 50002$

14. Kh Nouicer, Quantum-corrected black hole thermodynamics to all orders in the Planck length. Phys. Lett. B 626(2-3), 63 (2007). https://doi.org/10.1016/j.physletb.2006.12.072

15. Kh Nouicer, Black hole thermodynamics to all orders in the Planck length in extra dimensions. Class. Quantum Gravity 24(23), 5917 (2007). https://doi.org/10.1088/0264-9381/24/23/014

16. W. Kim, E.J. Son, M. Yoon, Thermodynamics of a black hole based on a generalized uncertainty principle. J. High Energy Phys. 2008, 035 (2008). https://doi.org/10.1088/1126-6708/2008/01/035

17. K. Nouicer, Quantum gravity effects from generalized uncertainty principle, in Conference Paper (2009). https://www.researchgate. net/publication/280384349

18. Y.C. Ong, An effective black hole remnant via infinite evaporation time due to generalized uncertainty principle. J. High Energy Phys. 2018, 95 (2018). https://doi.org/10.1007/JHEP10(2018)195

19. M. Faizal, Deformation of second and third quantization. Int. J. Mod. Phys. A 30(9), 1550036 (2015). https://doi.org/10.1142/ S0217751X15500360

20. Y.C. Ong, Generalized uncertainty principle, black holes, and white dwarfs: a tale of two infinities. J. Cosmol. Astropart. Phys. 09, 015 (2015). https://doi.org/10.1088/1475-7516/2018/09/015

21. A.M. Diab, A.N. Tawfik, A possible solution of the cosmological constant problem based on minimal length uncertainty and GW170817 and PLANCK Observations. arXiv:2005.03999v1

22. M. Khodadi, K. Nozari, E.N. Saridakis, Emergent universe in theories with natural UV cutoffs. Class. Quantum Gravity 35(1), 015010 (2018). https://doi.org/10.1088/1361-6382/aa95aa

23. L.N. Chang, Z. Lewis, D. Minic, T. Takeuchi, On the minimal length uncertainty relation and the foundations of string theory. Adv. High Energy Phys. 2011, 493514 (2011). https://doi.org/10. 1155/2011/493514

24. S. Hossenfelder, Minimal length scale scenarios for quantum gravity. Living Rev. Relativ. 16, 2 (2013). https://doi.org/10.12942/ 1rr-2013-2

25. A. Tawfik, A. Diab, Generalized uncertainty principle: approaches and applications. Int. J. Mod. Phys. D 23(12), 1430025 (2014). https://doi.org/10.1142/S0218271814300250

26. A. Tawfik, A. Diab, Review on generalized uncertainty principle. Rep. Prog. Phys. 78(12), 126001 (2015). https://doi.org/10.1088/ $0034-4885 / 78 / 12 / 126001$

27. G. Veneziano, A stringy nature needs just two constants. Europhys. Lett. 2(3), 199 (1986). https://doi.org/10.1209/0295-5075/2/3/006

28. D. Amati, M. Ciafaloni, G. Veneziano, Superstring collisions at Planckian energies. Phys. Lett. B 197(1-2), 81 (1987). https://doi. org/10.1016/0370-2693(87)90346-7

29. D. Amati, M. Ciafaloni, G. Veneziano, Can space-time be probed below the string size? Phys. Lett. B 216(1-2), 41 (1989). https:// doi.org/10.1016/0370-2693(89)91366-X

30. D.J. Gross, P.F. Mende, The high-energy behavior of string scattering amplitudes. Phys. Lett. B 197(1-2), 129 (1987). https://doi. org/10.1016/0370-2693(87)90355-8 
31. D.J. Gross, P.F. Mende, String theory beyond the Planck scale. Nucl. Phys. B 303(3), 407 (1988). https://doi.org/10.1016/ 0550-3213(88)90390-2

32. K. Konishi, G. Paffuti, P. Provero, Minimum physical length and the generalized uncertainty principle in string theory. Phys. Lett. B 234(3), 276 (1990). https://doi.org/10.1016/ 0370-2693(90)91927-4

33. M. Maggiore, A generalized uncertainty principle in quantum gravity. Phys. Lett. B 304(1-2), 65 (1993). https://doi.org/10.1016/ 0370-2693(93)91401-8

34. F. Scardigli, Generalized uncertainty principle in quantum gravity from micro-black hole Gedanken experiment. Phys. Lett. B 452(12), 39 (1999). https://doi.org/10.1016/S0370-2693(99)00167-7

35. Mu-In Park, The generalized uncertainty principle in (A)dS space and the modification of Hawking temperature from the minimal length. Phys. Lett. B 659(3), 698 (2008). https://doi.org/10.1016/ j.physletb.2007.11.090

36. F. Scardigli, Generalized uncertainty principle, extra dimensions and holography. Class. Quantum Gravity 20(18), 3915 (2003). https://doi.org/10.1088/0264-9381/20/18/305

37. A. Kempf, G. Mangano, R.B. Mann, Hilbert space representation of the minimal length uncertainty relation. Phys. Rev. D 52(2), 1108 (1995). https://doi.org/10.1103/PhysRevD.52.1108

38. A.F. Ali, S. Das, E.C. Vagenas, Discreteness of space from the generalized uncertainty principle. Phys. Lett. B 678(5), 497 (2009). https://doi.org/10.1016/j.physletb.2009.06.061

39. B. Majumder, Effects of GUP in quantum cosmological perfect fluid models. Phys. Lett. B 699(5), 315 (2011). https://doi.org/10. 1016/j.physletb.2011.04.030

40. B. Majumder, Quantum black hole and the modified uncertainty principle. Phys. Lett. B 701(4), 384 (2011). https://doi.org/10. 1016/j.physletb.2011.05.076

41. B. Majumder, The generalized uncertainty principle and the Friedmann equations. Astrophys. Space Sci. Lett. 336, 331 (2011). https://doi.org/10.1007/s10509-011-0815-6

42. S. Giardino, V. Salzano, Cosmological constraints on GUP from modified Friedmann equations. Eur. Phys. J. C 81, 110 (2021). https://doi.org/10.1140/epjc/s10052-021-08914-2

43. A. Kempf, Mode generating mechanism in inflation with a cutoff. Phys. Rev. D 63(8), 083514 (2001). https://doi.org/10.1103/ PhysRevD.63.083514

44. S.F. Hassan, M.S. Sloth, Trans-Planckian effects in inflationary cosmology and the modified uncertainty principle. Nucl. Phys. B 674(1-2), 434 (2003). https://doi.org/10.1016/j.nuclphysb.2003. 09.041

45. A. Ashoorioon, A. Kempf, R.B. Mann, Minimum length cutoff in inflation and uniqueness of the action. Phys. Rev. D 71(2), 023503 (2005). https://doi.org/10.1103/PhysRevD.71.023503

46. A. Kempf, L. Lorenz, Exact solution of inflationary model with minimum length. Phys. Rev. D 74(10), 023503 (2006). https://doi. org/10.1103/PhysRevD.74.103517

47. B. Vakili, H.R. Sepangi, Cosmology with minimal length uncertainty relations. Int. J. Mod. Phys. D 18(7), 1059 (2009). https:// doi.org/10.1142/S0218271809014935

48. A.N. Tawfik, A.M. Diab, Generalized uncertainty principle and recent cosmic inflation observations. Electron. J. Theor. Phys. 12(32), 9 (2015)

49. A. Mohammadi, A. Farag Ali, T. Golanbari, A. Aghamohammadi, Kh Saaidi, M. Faizal, Inflationary universe in the presence of a minimal measurable length. Ann. Phys. 385, 214 (2017). https:// doi.org/10.1016/j.aop.2017.08.008

50. K. Atazadeh, F. Darabi, Einstein static universe from GUP. Phys. Dark Universe 16, 87 (2017). https://doi.org/10.1016/j.dark.2017. 04.008
51. M. Moumni, A. Fouhal, Minimal length effects on Friedmann equations. Int. J. Mod. Phys. A 35(2-3), 2040043 (2015). https://doi. org/10.1142/S0217751X20400436

52. M. Moussa, Effect of generalized uncertainty principle on mainsequence stars and white dwarfs. Adv. High Energy Phys. 2015, 343284 (2015). https://doi.org/10.1155/2015/343284

53. R. Rashidi, Generalized uncertainty principle and the maximum mass of ideal white dwarfs. Ann. Phys. 374, 434 (2016). https:// doi.org/10.1016/j.aop.2016.09.005

54. P. Jizba, H. Kleinert, F. Scardigli, Uncertainty relation on a world crystal and its applications to micro black holes. Phys. Rev. D 81(8), 084030 (2010). https://doi.org/10.1103/PhysRevD.81.084030

55. R.N. Costa Filho, J.P.M. Braga, J.H.S. Lira, J.S. Andrade Jr., Extended uncertainty from first principles. Phys. Lett. B 755, 367 (2016). https://doi.org/10.1016/j.physletb.2016.02.035

56. S. Kouwn, Implications of minimum and maximum length scales in cosmology. Phys. Dark Universe 21, 76 (2018). https://doi.org/ 10.1016/j.dark.2018.07.001

57. L. Perivolaropoulos, Cosmological horizons, uncertainty principle, and maximum length quantum mechanics. Phys. Rev. D 95(10), 103523 (2017). https://doi.org/10.1103/PhysRevD.95.103523

58. L. Perivolaropoulos, Primordial power spectra of cosmological fluctuations with generalized uncertainty principle and maximum length quantum mechanics. Phys. Rev. D 100(12), 123527 (2019). https://doi.org/10.1103/PhysRevD.100.123527

59. S. Jalalzadeh, M. Ali Gorji, K. Nozari, Deviation from the standard uncertainty principle and the dark energy problem. Gen. Relativ. Gravit. 46(1), 1632 (2014). https://doi.org/10.1007/ s10714-013-1632-8

60. A. Vilenkin, Approaches to quantum cosmology. Phys. Rev. D 50(4), 2581 (1994). https://doi.org/10.1103/PhysRevD.50.2581

61. B.F. Shutz, Perfect fluids in general relativity: velocity potentials and a variational principle. Phys. Rev. D 2(12), 2762 (1970). https:// doi.org/10.1103/PhysRevD.2.2762

62. B.F. Shutz, Hamiltonian theory of a relativistic perfect fluid. Phys. Rev. D 4(12), 27262 (1970). https://doi.org/10.1103/PhysRevD.4. 3559

63. F.G. Alvarenga, N.A. Lemos, Dynamical vacuum in quantum cosmology. Gen. Relativ. Gravit. 30(5), 681 (1998). https://doi.org/ 10.1023/A: 1018896900336

64. B.S. DeWitt, Quantum theory of gravity. I. The canonical theory. Phys. Rev. 160, 1113 (1967). https://doi.org/10.1103/PhysRev. 160.1113

65. P. Pedram, Generalized uncertainty principle and the conformally coupled scalar field quantum cosmology. Phys. Rev. D 91(6), 063517 (2015). https://doi.org/10.1103/PhysRevD.91.063517

66. P. Pedram, A higher order GUP with minimal length uncertainty and maximal momentum. Phys. Lett. B 714(2-5), 317 (2012). https://doi.org/10.1016/j.physletb.2012.07.005

67. P. Pedram, A higher order GUP with minimal length uncertainty and maximal momentum II: applications. Phys. Lett. B 718(2), 638 (2012). https://doi.org/10.1016/j.physletb.2012.10.059

68. G. Amelino-Camelia, Relativity in spacetimes with short-distance structure governed by an observer-independent (Planckian) length scale. Int. J. Mod. Phys. D 11(1), 35 (2002). https://doi.org/10. 1142/S0218271802001330

69. G. Amelino-Camelia, Special treatment. Nature 418(1), 34 (2002). https://doi.org/10.1038/418034a

70. J. Magueijo, L. Smolin, Lorentz invariance with an invariant energy scale. Phys. Rev. Lett. 88(19), 190403 (2002). https://doi.org/10. 1103/PhysRevLett.88.190403

71. P. Pedram, On the boundary conditions in deformed quantum mechanics with minimal length uncertainty. Adv. High Energy Phys. 2013, 853696 (2013). https://doi.org/10.1155/2013/853696 
72. C.A. Mead, Possible connection between gravitation and fundamental length. Phys. Rev. 135, B849 (1964). https://doi.org/10. 1103/PhysRev.135.B849

73. H. Shababi, W.S. Chung, A new type of GUP with commuting coordinates. Mod. Phys. Lett. A 35(6), 2050018 (2020). https:// doi.org/10.1142/S0217732320500182

74. S. Detournay, C. Gabriel, P. Spindel, About maximally localized states in quantum mechanics. Phys. Rev. D 66(12), 125004 (2002). https://doi.org/10.1103/PhysRevD.66.125004
75. D. Lovelock, The Einstein tensor and its generalizations. J. Math. Phys. 12(3), 498 (1971). https://doi.org/10.1063/1.1665613

76. F. Bianchini et al., Constraints on cosmological parameters from the $500 \mathrm{deg}^{2}$ SPTPOL lensing power spectrum. Astrophys. J. 888(2), 119 (2020). https://doi.org/10.3847/1538-4357/ab6082

77. G. Efstathiou, S. Gratton, The Einstein tensor and its generalizations. Mon. Not. R. Astron. Soc. 496(1), L91 (2020). https://doi. org/10.1093/mnrasl/slaa093

78. K. Zeynali, F. Darabi, H. Motavalli, Multi-dimensional cosmology and GUP. J. Cosmol. Astropart. Phys. 12, 033 (2012). https://doi. org/10.1088/1475-7516/2012/12/033 